\title{
Directed evolution of CRISPR-Cas9 to increase specificity
}

Building upon previous CRISPR
research and gene editing
methods found in nature,
Dr Lee and colleagues from
ToolGen have developed a
method of screening multiple
Cas9 variants. Their method
seeks to quickly and effectively
screen many mutations
of Cas9 to find one which
possesses maximised on-
target activity and minimised
off-target activity. After
successful development and
implementation of the system,
Sniper-Screen, a suitable Cas9
variant was found: Sniper-Cas9.

ur DNA controls many
elements of our lives and
any fault in our DNA can p until now, it has been very difficut to fix faults in our DNA; however

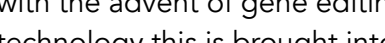
the realm of the possible.

USING CRISPR TO EDIT GENES One such gene editing technology is CRISPR: Clustered Regularly Interspaced Short Palindromic Repeats. Already gaining significant attention amongst scientists, CRISPR is a simple yet powerful tool for genome editing. CRISPR allows researchers to easily alter DNA sequences and consequen modify gene function.

Whilst gene editing technology does scientific communities, there anongst examples of similar genome
The CRISPR Cas9 genome
edititing tool was adapted editing tool was
from a naturally from a naturally
occurring system
found in bacteria.

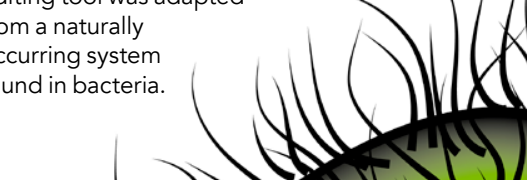
diting systems in nature.
One of the most versat and precise methods of genome editing currently available
is CRISPR Cas9 is CRISPR Cas9 which was adapted from a naturally found in bacteria.

NATURALLY OCCURRING CRISPR CAS9

CRISPR CAS9
In nature, CRISPR Cas 9 acts as an immune system for bacteria against invading viruses. Naturally occurring
Cas9 serves as an excellent template for the development of a suitable it does have some flaws. Cas 9 whic demonstrates high specificity to the viruses it can protect agains is disadvantageous as viruses are prone to spontaneous mutation. If the Cas9 is highly specific then it will not be able to cope with viral mutation, however Cas9 with promiscuous specificity could continue to be effective. For this reason, it is considered highly likely that Cas9 sequences found in nature show promiscuous specificities.

Being able to screen a large number of mutations for a variant of Cas9 with optimal amount of specificity to develop a therapy to modry human gene function was the aim of the latest and his colleagues at ToolGen.

\section{THE RESEARCH TEAM}

Dr Lee was UK representative for the 33rd International Chemistry Olympiads and obtained a silver medal. He went from Queens' College, Cambridge and achieved his PhD from Stanford University. Following his studies, he joined Samsung Advanced Institute of Technology (SATT), supporting a target discovery project for Samsung Biologics. Professor Lee then joined start-up company Toolgen where of is currently Research Head of Platform Development.

In this latest work Dr Lee was supported by colleagues from ToolGen, Joonsun

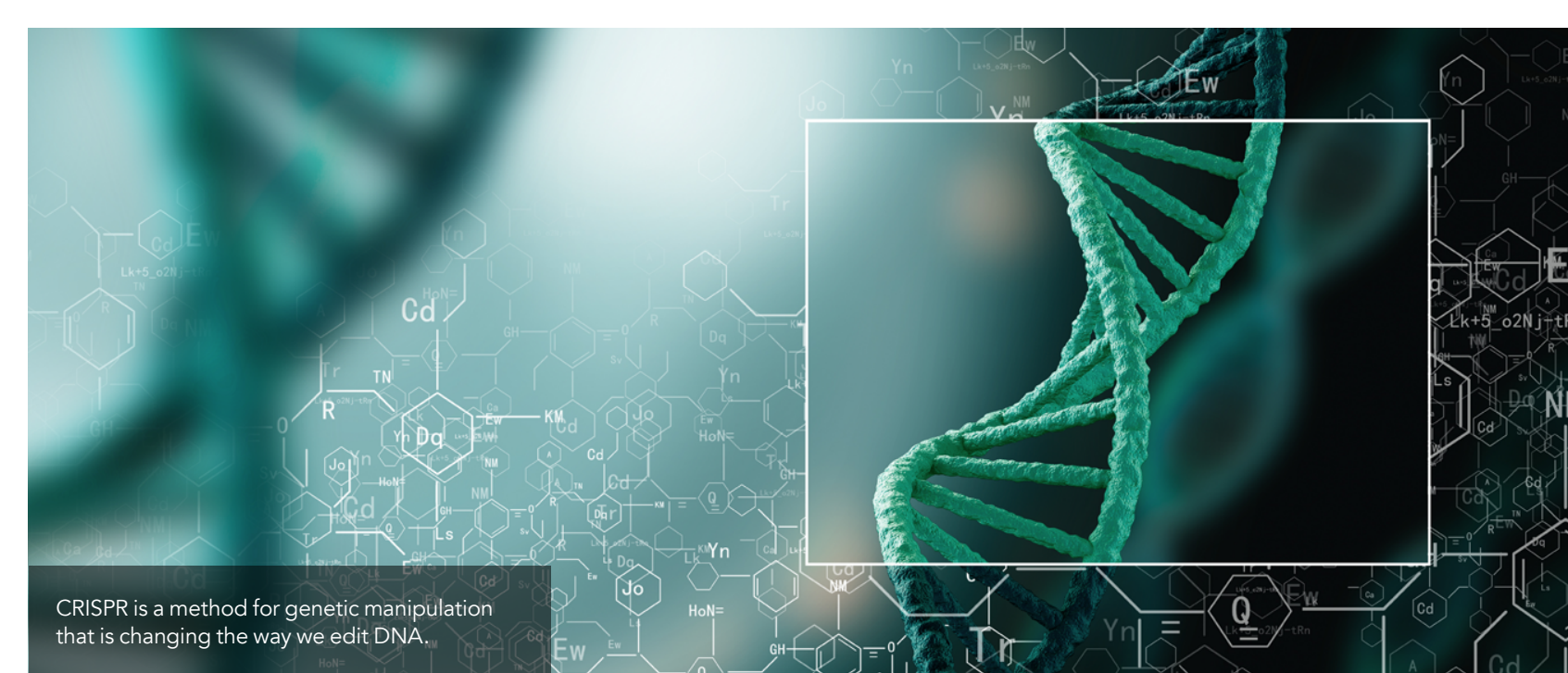

Lee, Min-hee Jung and Eulhwan Jeong and by Professor Jin-Soo Kim. Grants Ministry of Scie for alic Science, and Nation of Korea, funded by the Korean government supported this research.

BUILDING ON PREVIOUS RESEARCH In this study, the team developed a method of screening a broad range of mutated Cas9 variants to seek one with the best characteristics for use in human therapy. The ideal characteristics include high levels of "on-target" desirable effects and low levels of "off-target" effects. This means a variant with high specificity will be sought for use in human healthcare. This contradicts the natura tendenicy of Cas\% to have promiscuous As Cas? modifies genes it is important that researchers can control which genes will be edited and understand what will happen to them before implementing a therapy.

In order to undertake this research, the ToolGen researchers needed to build upon previous work exploring several elements of different methods which could be used to minimise the undesirable effects of wild type Cas9.

For example, previous research has shown that using truncated single guide RNAs (sgRNAs) to guide gene higher specificity be used to achieve RNA meccifity. sgRNA is a single designed shor crRNA sequence fused

to the scaffold tracrRNA sequence. The team also used research which show hat changing the method of ribonucleoprotein (RNP) format allows higher specificity to be achieved.

To be able to screen the broadest variety of Cas9 variants possible the team built upon previous research antages over a yeast system because of the faster doubling efficiencies of E. coli.

Many groups have previously reported that Cas9 variants engineered using the design to debilitate the non-specific interaction between Cas9 and the substrate exhibit low on-target activities. Although

In nature, CRISPR Cas9 acts as an immune system for bacteria against invading viruses.

of Cas9. For example, in Streptococcus minimising off-target activities is pyogenes Cas9 (SpCas9), specific pyegerid the method of screening was explored. in this ead of screaning was explons with the highest specificity were identified using either a yeast or an $E$.
coli screening system. Previous work has shown that an E. coli screening exremely important, it is equally essential that on-target activites are simply not work.

Having reviewed and evaluated the previous research, the team at ToolGe developed an $E$ coli based directed evolution system to screen randomly

Selection Scheme: Sniper-Screen can select Cas9 variants with high specificity

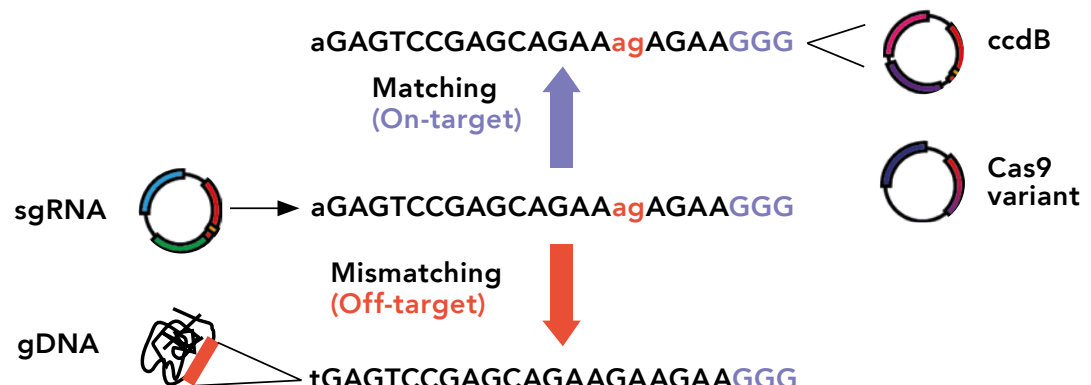


mutagenised Cas 9 variants whils minimised anget activity was was increased.

\section{USING SNIPER-SCREEN}

Lee and the Toolgen team

chose to name their method

Sniper-Screen, to reflect the high

level of accuracy their method can

achieve in searching for the target Cas 9 and retained on-target activity.

By combining both positive screening systems and negative screening

systems, the on-target and off-target effects of the Cas 9 can be evolved spontaneously. Sniper-Screen is based on three different plasmids and a gene of interest integrated into the
E. coli genome. The complex process required to study these variants required several steps.

Importantly, in their method Cas9 variants are expressed under the CMV-PltetO1 dual-promoter system of a low-copy number plasmid so that candidates identified in E. col can be tested in mammalian cells without the need for sub-cloning. This helps to ensure the method can be completed as efficiently as possible.

The gene of interest is then introduced into the E. coli genome using the Tn7 transposon system. The sgRNA plasmid, which contains a temperaturean sgRNA to guide development targeting the gene of interest, howeve the sgRNA and gene of interest sequences are not perfectly matched.

A perfectly matched sgRNA target site exists on a third plasm the $c c d B$ gene, which encodes a lethal product that poisons the enzyme gyrase and facilitates double strand breaks. In sniper-screen, cells expressing Cas? variants with high off-target activities are removed because double-strand breaks are introduced into the mismatched site located in the genomic DNA. At the same time, cells expressing Cas? variants with low on-target activities are also removed because oflethal

Increasing specificity of CRISPR-Cas9: Towards SNP differentiation Cell

$\rightarrow$
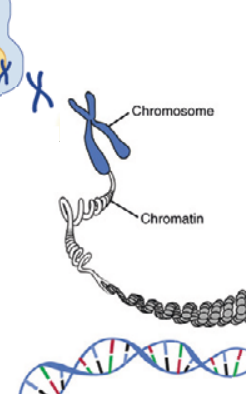

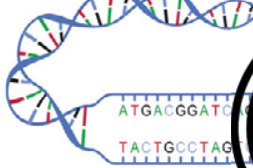

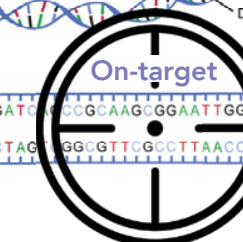

Using Sniper-Screen Lee and colleagues successfully identified a Cas9 variant with three mutations, called Sniper-Cas9...

level of the Cas 9 variants can be changed by altering the concentration of anhydrotetracycline (ATC), which adjusts the selection force.

with three mutations, called Sniper-Cas 9 which shows wild type levels of on-target activities and reduced off-target activities. Sniper-Cas9 can achieve even higher specificity ratios by using truncated sgRNAs or RNP-based delivery rather

The ToolGen team calculated that locating the mismatched sgRNA target site in the genomic DNA rather than on The ToolGen team used all resources at a plasmid would increase the sensitivity of the system. The advantage of this approach is that there is only one genomic site, whereas there would a target site, within a single E, colicell. $\begin{array}{ll}\text { Using Sniper-Screen Lee and colleagues } & \begin{array}{l}\text { ensure the momentum behind these } \\ \text { successfully identified a Cas9 variant }\end{array}\end{array}$ their disposal including a comprehensive review of the characteristics of wild type Cas9 and built upon previous result CRISPR gene technology a rapidly developing field and the work conducted by Lee and team will ensure the momentum behind these

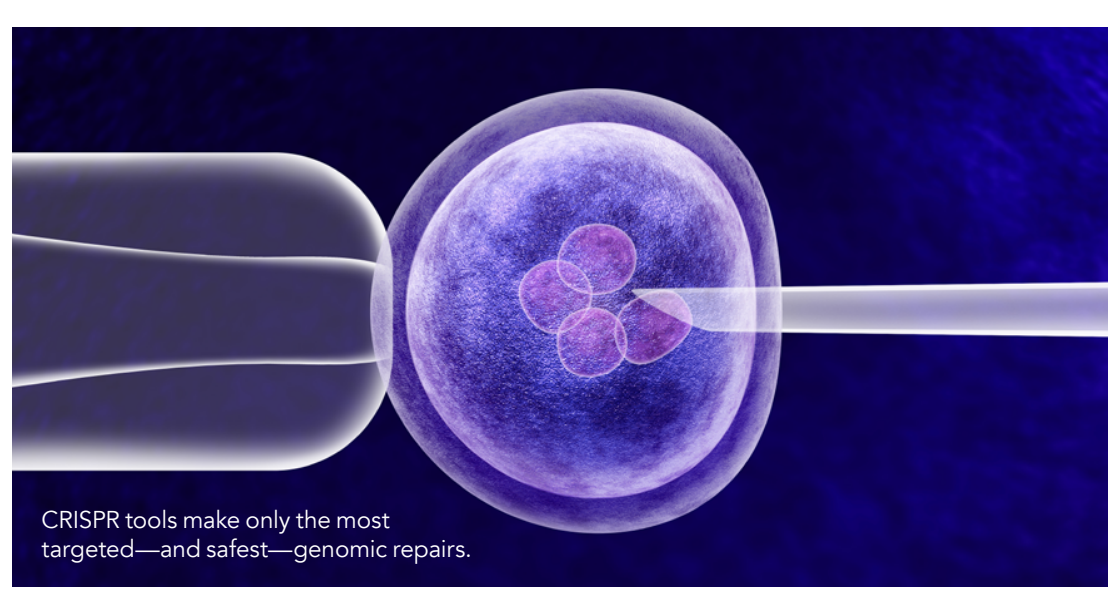
Jungjoon Lee

Research Objectives

Founded in 1999, ToolGen develops genome editing fechnology, and specialises in CRISPR/Cas9 systems.

\section{Detail}

Jungjoon Lee

Byuksan Digital Valley 6-cha,

Gasan Digital 1-ro,

Geumchun-gu,

Seoul, Korea

Bio

Dr Jungjoon Lee was UK representative for 33rd International Chemistry Olympiads and obtained a silver medal. He obtained his undergraduate degree at Queens' College, Cambridge and PhD from Stanford University. He then worked at Samsung Advanced Univist 列

\section{Funding}

This research was supported by grants from the Institute for Basic Science (IBS-RO21-DI) to J.-S.K., and Ministry and Nationa Research Foundation of Kor) (NRF) funded by the Korea government (MSIT) (grant 2017M3A9B4061404 and 2018M3A9H3020844) to J.K.L.

\section{Collaborator}

Professor Jin-soo Kim

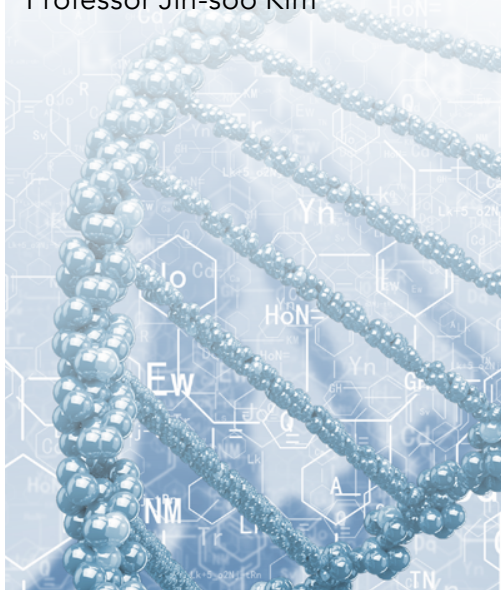

\section{References}

Lee, J., Jung, M.H., Jeong, E. and Lee, J.K., (2019). Using Sniper-Cas9 to Minimize Off-target Effects of CRISPR-Cas Without the Loss of On-target Activity Via Directed Evolution. JoVE (144), p.e59202. doi: 10.3791/59202

Lee, J.K., Jeong, E., Lee, J. et al. Directed evolution of mun 9, 3048 (2018) doi:10.1038/s41467-018-05477-x

\section{Personal Response}

CRISPR is a rapidly developing field with huge see coming next in this exciting are of ro you expect to

II With CRISPR, scientists can modify target genes with high specificity. Inducing double strand breaks is just the beginining. Other kinds of modifications are possible by simply fusing various enzymes with other functions developed in the near future.

\section{䝙ToolGen}

